\title{
Enhancing the Efficiency of Compact Patch Antennas Composed of Split-Ring Resonators by Using Lumped Capacitors
}

\author{
Elena Pucci, Eva Rajo-Iglesias, Senior Member, IEEE, Malcolm Ng Mou Kehn, Member, IEEE, and \\ Oscar Quevedo-Teruel, Member, IEEE
}

\begin{abstract}
A new type of small patch antenna with low profile and enhanced radiation efficiency is proposed in this letter. The antenna is realized with a double layer of low-permittivity material (polypropylene, $\varepsilon_{\mathrm{r}}=\mathbf{2 . 2}$ ). The lower layer is used for the feeding of the antenna, and split ring resonators (SRRs) are printed on top of the upper layer acting as radiating elements. The compactness is provided by shorting the rings to the ground plane with two metal pins. Although this antenna presented initially a dual band of operation, it has been demonstrated how the use of a lumped capacitor in the inner ring can increase the total radiation efficiency of the antenna performing a single-band response. Therefore, when the two original operation frequency bands coincide, a manufactured prototype of the antenna demonstrated a measured radiation efficiency of $73 \%$ that can be provided at the operation frequency of $1.29 \mathrm{GHz}$.
\end{abstract}

Index Terms-Electrically small antennas, lumped capacitor, microstrip patch antennas, radiation efficiency, split-ring resonators (SRRs).

\section{INTRODUCTION}

$\mathbf{T}$ HE DEVELOPMENT of wireless communications has raised the need for low-profile antennas with good radiation characteristics to be built inside mobile phones and wireless devices of the new generation. Microstrip patch antennas are typically used for this purpose, as they are realized in printed technology and they can be mounted directly in the circuit board [1]-[3]. On the other hand, their radiation efficiency is affected by the high-permittivity materials often used to obtain compact sizes and multifrequency performance [1], [4], [5].

In the last years, split-ring resonators (SRRs) have been used in antennas and microwaves components for several applica-

Manuscript received August 10, 2012; revised October 15, 2012; accepted November 07, 2012. Date of publication November 15, 2012; date of current version December 04, 2012. This work was supported in part by the National Science Council of Taiwan under Grant NSC 100-2221-E-009-142 and the Spanish Government under Project TEC2010-20841-C04-01.

E. Pucci is with the Department of Signals and Systems, Chalmers University of Technology, Gothenburg 41296, Sweden (e-mail: elena.pucci@chalmers.se).

E. Rajo-Iglesias is with the Department of Signal Theory and Communication, University Carlos III of Madrid, 28911 Leganes (Madrid), Spain (e-mail: eva@tsc.uc3m.es)

M. Ng Mou Kehn is with the Department of Electrical Engineering, National Chiao Tung University, Hsinchu 30010, Taiwan (e-mail: malcolm.ng@ieee. org).

O. Quevedo-Teruel is with the Department of Electronic Engineering and Computer Science, Queen Mary University of London, London E1 4NS, U.K. (e-mail: oquevedo@eecs.qmul.ac.uk).

Color versions of one or more of the figures in this letter are available online at http://ieeexplore.ieee.org.

Digital Object Identifier 10.1109/LAWP.2012.2227662
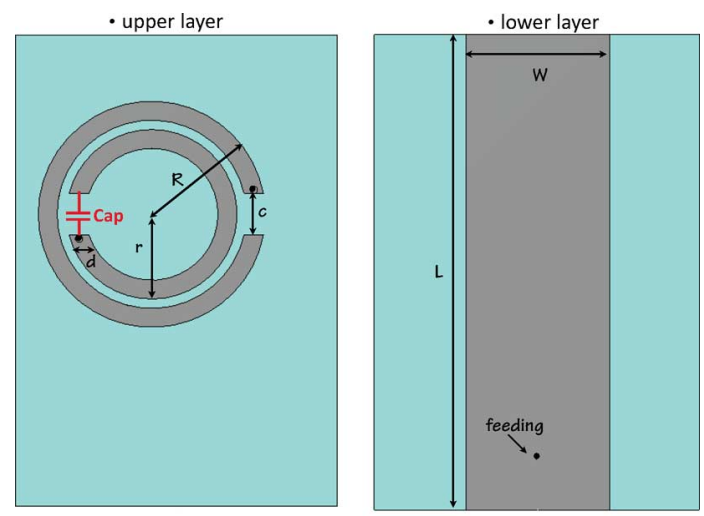

(a)

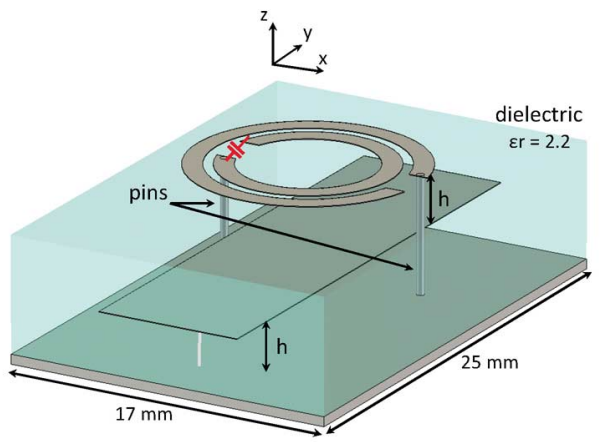

(b)

Fig. 1. (a) Top and (b) 3-D views of the proposed patch antenna with SRRs.

tions [6]-[8]. SRRs are well known in metamaterials since they can provide negative permeability that can create a stopband response at the resonant frequency [9], [10] and also produce new bands of operation [6], [11], [12]. In [13], a compact patch antenna is presented where the SRRs are used to create a dualband response. Finally, in [14], numerical results show how the frequency bands of the SRRs patch antenna can be moved by adding a lumped capacitor in the gap of the inner ring. The same approach is used in this letter to create a single-band patch antenna with higher radiation efficiency, for which an experimental validation is also provided. The idea of merging the two bands is similar to what has been used for decades in stacked patch antennas to increase bandwidth [2], [15], but here for the first time the focus is on the increase of the radiation efficiency.

\section{AntenNA DESIGN}

The proposed geometry, including the lumped capacitor in the gap of the inner ring, is presented in Fig. 1. The antenna has 


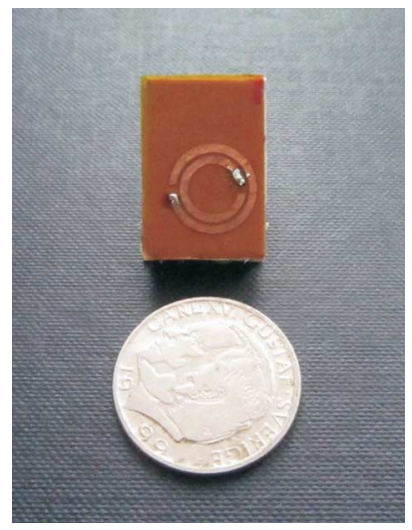

Fig. 2. Manufactured prototype.

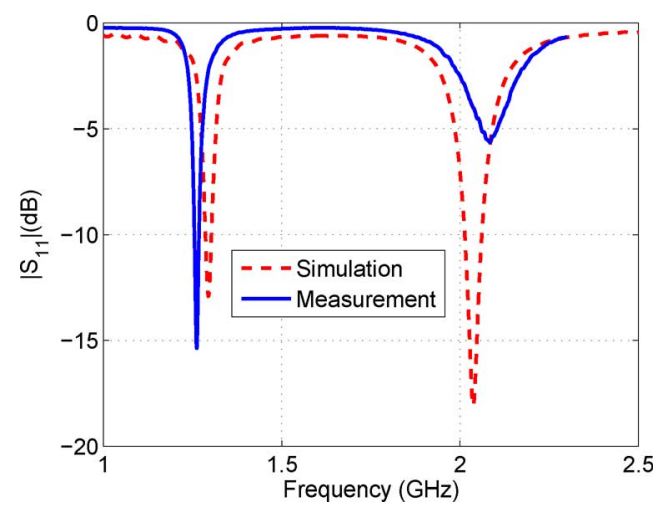

Fig. 3. Measurement and simulation reflection coefficients.

two layers, both of thickness $h=3 \mathrm{~mm}$. The lower layer is used to provide the feeding through a line of length $L=25 \mathrm{~mm}$ and width $W=7.3 \mathrm{~mm}$ on a polypropylene dielectric with permittivity $\varepsilon_{\mathrm{r}}=2.2$. This line is fed with a standard SMA coaxial connector. The radiating elements are two concentric SRRs with oppositely facing gaps that are printed on the upper layer with the same substrate material and thickness as the lower one. They are short-circuited to the ground plane by two metal pins attached to an end of each ring near its gap as shown in Fig. 1. The dimensions of the rings are chosen as follows: inner and outer ring radius $r=4.5 \mathrm{~mm}$ and $R=6 \mathrm{~mm}$, respectively, gap $c=1.4 \mathrm{~mm}$, and ring width $d=1 \mathrm{~mm}$. The dielectric has been chosen with a low permittivity in order to not affect the radiation efficiency, otherwise reduced when using materials with higher permittivity. On the other hand, in order to provide a compact design, the two pins connected to the end of each ring and short-circuited to the ground plane behave as inductances, similarly to planar inverted-F antennas (PIFAs) [13].

The prototype, first considered without capacitance, is shown in Fig. 2, and in Fig. 3 measurement and simulation results of the $S_{11}$ are presented. Simulations were carried out with the commercial software CST Microwave Studio [16]. Simulated and measurement results agree well, showing the double-band response due to the two SRRs. The radiation efficiency for this antenna has been measured in a reverberation chamber [17], and it is shown in Fig. 4. The total radiation efficiency (solid curve) includes the mismatch factor, whereas the radiation efficiency (dashed curve) is the antenna efficiency without loss contribu-

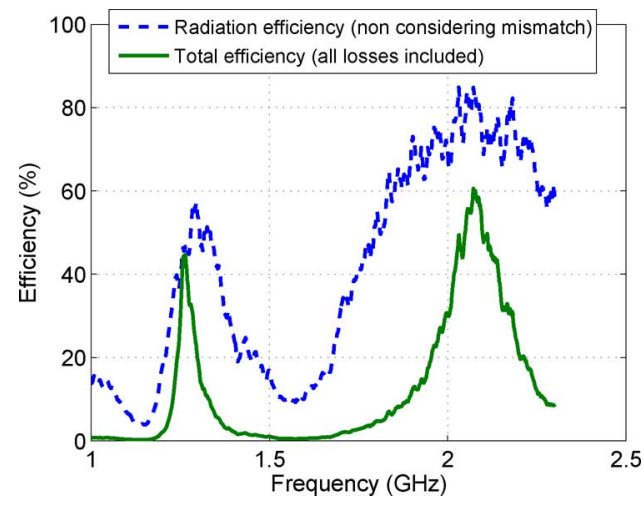

Fig. 4. Measured radiation efficiency and total radiation efficiency (including mismatch factor).

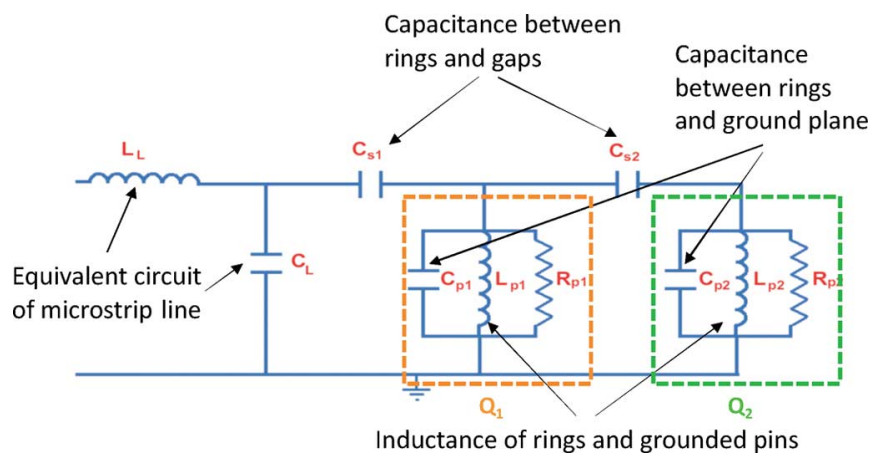

Fig. 5. Circuit model of the antenna.

tion due to the mismatch. The total efficiency at the center of the lower frequency band at $1.26 \mathrm{GHz}$ is about $45 \%$, and in this case the radiation efficiency and total efficiency almost coincide at this operation frequency since the antenna is well matched. At the upper-band center frequency $(2.08 \mathrm{GHz})$, the efficiency is around $80 \%$ if the reflection losses are not taken into account.

\section{EFFECT OF LUMPED CAPACITORS}

By adding a capacitor in between the gap of the inner ring, as shown in Fig. 1, the upper frequency band (which is defined by the inner ring) shifts down until, for a certain value of the capacitance, it meets the lower band, thus becoming a single-band antenna. The resulting efficiency will be larger for this "single-band" antenna, compared to the dual-band case, since it will be an effective contribution from both rings to the radiation. The reason for the increase of radiation efficiency with the use of the lumped capacitor may be two-pronged conveyed as a qualitative argumentation and a somewhat quantitative explanation. Qualitatively first, by using lumped capacitors to shift the upper band down toward the lower one and thereby merging them, we obtain two resonances at the same frequency. In a more mathematical (quantitative) way, the equivalent circuit model of the antenna is represented as a parallel resonant circuit comprising two $R L C$ resonators in parallel, each pertaining to one of the two rings, as shown in [13] and represented in Fig. 5.

It is also first acknowledged that the $Q$-factor is inversely related to the radiation efficiency, which can be conceived intuitively by the mere definition of the $Q$-factor. Then, from the 


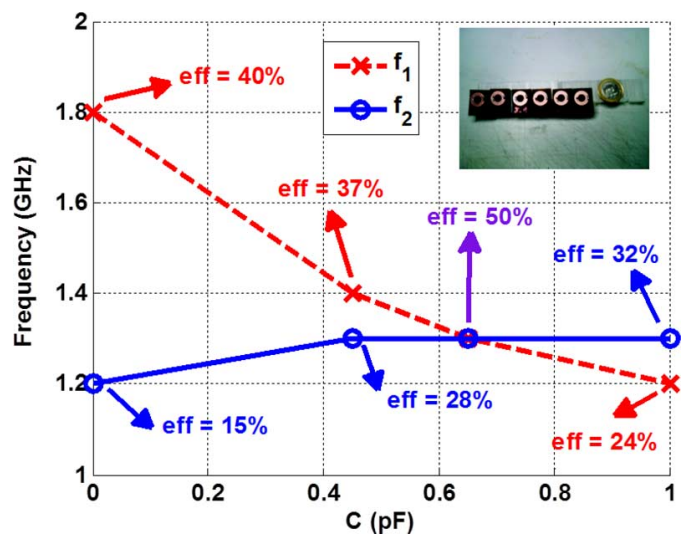

Fig. 6. Measured operation frequencies when a capacitor is placed in the gap of the inner ring. For each measurement, it is also indicated the radiation efficiency $R=6.1 \mathrm{~mm}, r=4.6 \mathrm{~mm}, c=2.2 \mathrm{~mm}, d=1 \mathrm{~mm}, h=2 \mathrm{~mm}$, and $\epsilon_{\mathrm{r}}=2.7$.

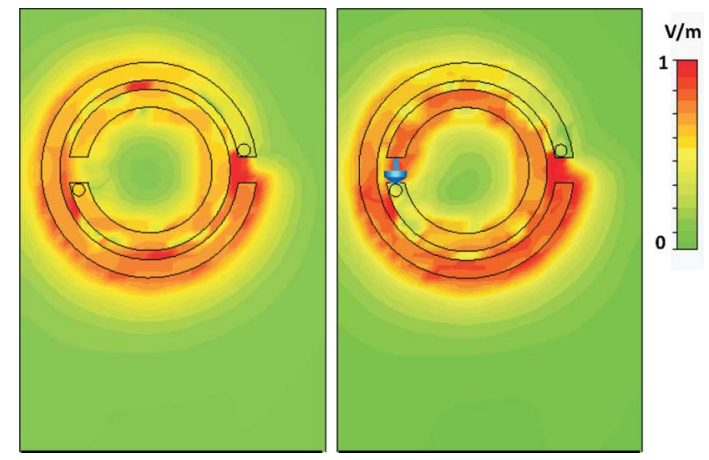

Fig. 7. Simulated electric field distribution for the antenna (right) with and (left) without capacitor of $0.82 \mathrm{pF}$ at the lower frequency of operation.

well-known expression of the $Q$-factor of a parallel resonant circuit: $Q=\omega_{0} R C$ where $\omega_{0}$ is the resonant frequency, raising the capacitance in the gap of the inner ring, which is responsible for the higher band, leads to a higher $Q$ of that upper band, thus a reduced efficiency. However, at the same time, although the $Q$ of the outer (larger) ring $\left(Q_{1}\right)$ on its own remains unchanged (no lumped capacitor added to it), the effective (total) $Q_{\mathrm{tot}}=Q_{1} \| Q_{2}$ of the two resonant circuits in parallel is predominated by the smaller of the two individual $Q$ values if their difference is large, which is that of the upper band. Hence, although the $Q_{2}$ of the upper band increases with the inclusion of the capacitor, it is still much smaller than the individual $Q_{1}$ of the outer ring. As such, when the two bands merge, although the efficiency of the upper band falls, that of the lower band increases from its original value before the merging due to the effective fall in the overall $Q$ at that common frequency. This effect is shown in Fig. 6 for the antenna (with $R=6.1 \mathrm{~mm}$, $r=4.6 \mathrm{~mm}, c=2.2 \mathrm{~mm}, d=1 \mathrm{~mm}, h=2 \mathrm{~mm}$, and $\left.\epsilon_{\mathrm{r}}=2.7\right)$ based on such resonance-merging for efficiency improvement. Here, the lower band $f_{2}$ (due to the outer ring) is fixed while the upper frequency $f_{1}$ (caused by the inner ring) is shifted toward it. The increase in the efficiency at $f_{2}$ is more than three times (15\% to $50 \%)$. Therefore, by merging two resonances, an increment of the radiation efficiency of the low frequency band can be achieved.

Coming back to the antenna whose dimensions were specified in Section II, in Fig. 7 the normalized electric field for both cases

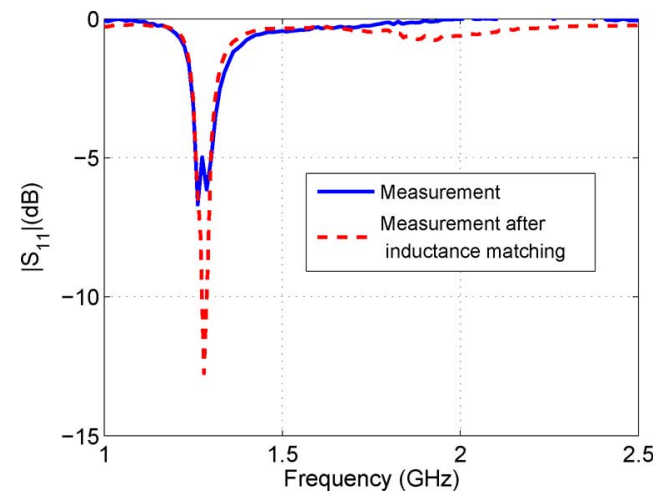

Fig. 8. Measured reflection coefficient when a lumped capacitor of $0.82 \mathrm{pF}$ is added in the inner ring; before and after series inductance matching.

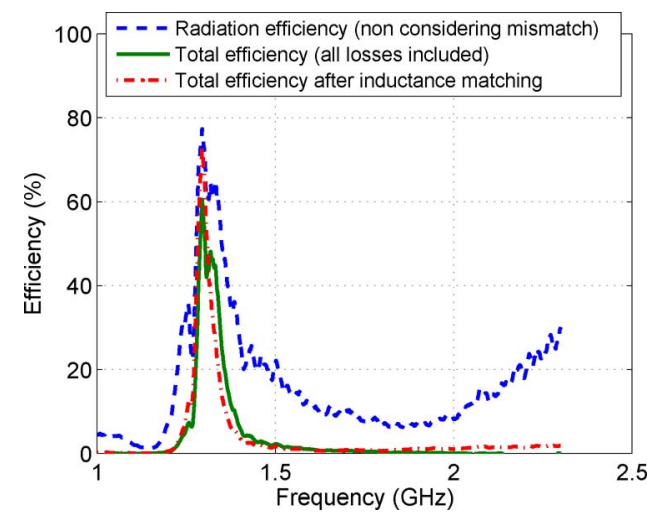

Fig. 9. Measured radiation efficiency and total radiation efficiency (including mismatch factor) for the prototype with capacitor; before and after series inductance matching.

(with and without capacitor) is illustrated at the lower frequency of operation at the $z$-plane immediately over the rings. In this figure, it is demonstrated how the inner ring contributes more actively to the radiation when the capacitor is introduced.

In order to validate this approach, a lumped capacitor was added in the gap of the inner conductor. A capacitance of $0.82 \mathrm{pF}$ was found to be enough in order to obtain the single-band response. Fig. 8 shows the measured $S_{11}$ (solid curve), and in Fig. 9, the radiation efficiency measured in a reverberation chamber is presented (dashed curve). The antenna is not completely matched at the operation frequency, and this can be seen from both the measured $S_{11}$ and total efficiency. However, this mismatch factor can be easily corrected with a series inductance as Fig. 8 shows (dashed curve), having a maximum total radiation efficiency of $73 \%$ at the operation frequency of $1.29 \mathrm{GHz}$, as finally Fig. 9 presents (dash-dotted curve).

The bandwidth of operation of the antenna (in terms of matching) is approximately $2 \%$, in which the radiation efficiency is higher than $70 \%$. These curves were obtained applying to the measurements a simulated model of the series inductance placed between the feeding (SMA connector) and the subsequent circuitry, just to show that the antenna matching can be easily achieved. This matching, which we call for simplicity "inductance matching" (because of the inclusion of a lumped series inductor to the circuit), can be realized in practice 


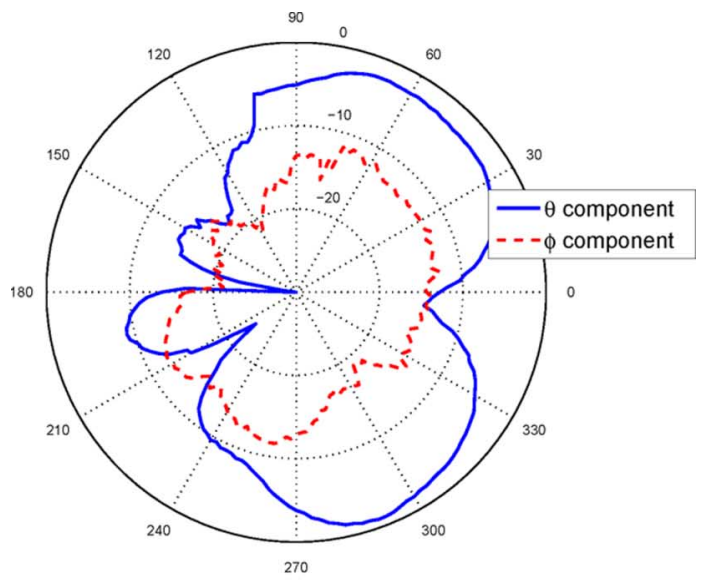

(a)

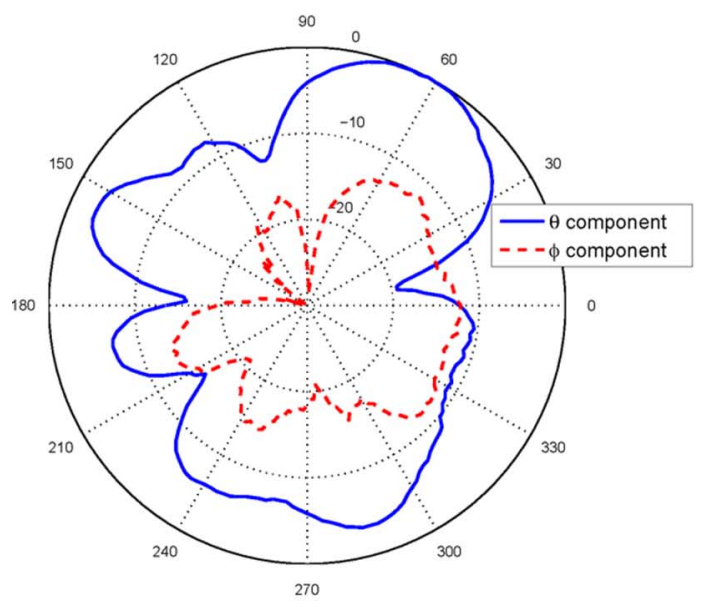

(b)

Fig. 10. Measured radiation pattern in the two main planes. (a) $y z$-plane. (b) $x z$-plane.

by using an external matching network, e.g., stubs or lumped inductors. In addition, it has also been verified by simulations that further miniaturization can be achieved by shortening the long side of the structure along the feed line (Fig. 1) to obtain a squarer, even more compact antenna without any adverse effects on the matching and efficiency performance. The size along the breadth is, however, limited by the outer ring.

Finally, the antenna radiation pattern has been measured, and it is shown in Fig. 10. As expected, the radiation pattern is quite isotropic. It is also similar to the (lower/upper) band of the PIFA studied in [13], with a null in the broadside direction due the geometry of the antenna.

\section{CONCLUSION}

A compact microstrip patch antenna with high radiation efficiency has been presented in this letter. First, a dual-band antenna with SRRs was designed and measured, showing two operation frequency bands at 1.26 and $2.08 \mathrm{GHz}$, created by the outer and inner rings, respectively. The measured radiation efficiency for this first prototype was found to be $45 \%$ (for the lower frequency) and about $80 \%$ (for the upper frequency, not including mismatch). Measurement results showed how the same antenna can perform better, when the two bands meet together at $1.29 \mathrm{GHz}$, by adding a capacitor of $0.82 \mathrm{pF}$. In this case, the measured radiation efficiency was found to be about $73 \%$, covering both requirements for compactness and good radiation characteristics typically difficult to acquire at the same time for small antennas. The total size of the designed antenna including the ground plane was $0.073 \lambda_{0}$ by $0.1 \lambda_{0}$. Finally, it was also verified by simulations that the size of the antenna can be further reduced by shortening its length without compromising the matching and efficiency.

\section{REFERENCES}

[1] J. R. James and P. S. Hall, Handbook of Microstrip and Printed Antennas. New York: Wiley, 1997.

[2] D. M. Pozar and D. H. Schaubert, Microstrip Antennas: The Analysis and Design of Microstrip Antennas and Arrays. New York: WileyIEEE Press, 1995.

[3] P. Bhartia, I. Bahl, R. Garg, and A. Ittipiboon, Microstrip Antenna Design Handbook. Norwood, MA: Artech House, 2000.

[4] Y. Zhou, C. C. Chen, and J. L. Volakis, "Dual band proximity-fed stacked patch antenna for tri-band GPS applications," IEEE Trans. Antennas Propag., vol. 55, no. 1, pp. 220-223, Jan. 2007.

[5] O. Quevedo-Teruel, E. Pucci, and E. Rajo-Iglesias, "Compact loaded PIFA for multifrequency applications," IEEE Trans. Antennas Propag., vol. 58, no. 3, pp. 656-664, Mar. 2010.

[6] O. Quevedo-Teruel, E. Rajo-Iglesias, and M. Ng Mou Kehn, "Numerical and experimental studies of split ring resonators loaded on the sidewalls of rectangular waveguides," Microw., Antennas Propag., vol. 3, no. 8 , pp. $1262-1270,2009$

[7] L. Jae-Gon and L. Jeong-Hae, "Suppression of spurious radiations of patch antenna using split ring resonators (SRRs)," in Proc. IEEE Antennas Propag. Soc. Int. Symp., 2005, vol. 2B, pp. 242-245.

[8] M. Duran-Sindreu, A. Velez, F. Aznar, G. Siso, J. Bonache, and F. Martin, "Applications of open split ring resonators and open complementary split ring resonators to the synthesis of artificial transmission lines and microwave passive components," IEEE Trans. Microw. Theory Tech., vol. 57, no. 12, pp. 3395-3403, Dec. 2009.

[9] J. B. Pendry, A. J. Holden, D. J. Robbins, and W. J. Stewart, "Magnetism from conductors and enhanced nonlinear phenomena," IEEE Trans. Microw. Theory Tech., vol. 47, no. 11, pp. 2075-2084, Nov. 1999.

[10] E. Rajo-Iglesias, O. Quevedo-Teruel, and M. Ng Mou Kehn, "Multiband SRR loaded rectangular waveguide," IEEE Trans. Antennas Propag., vol. 57, no. 5, pp. 1571-1575, May 2009.

[11] R. Marqués, J. Martel, F. Mesa, and F. Medina, "Left-handed media simulation and transmission of EM waves in sub-wavelength SRRloaded metallic waveguides," Phys. Rev. Lett., vol. 89, no. 18, pp. 183 901/1-4, Oct. 2002.

[12] S. Hrabar, J. Bartolic, and Z. Sipus, "Waveguide miniaturization using uniaxial negative permeability metamaterial," IEEE Trans. Antennas Propag., vol. 53, no. 1, pp. 110-119, Jan. 2005.

[13] O. Quevedo-Teruel, M. Ng Mou Kehn, and E. Rajo-Iglesias, "Dualband patch antennas based on short-circuited split ring resonators," IEEE Trans. Antennas Propag., vol. 59, no. 8, pp. 2758-2765, Aug. 2011.

[14] O. Quevedo-Teruel, M. Ng Mou Kehn, E. Pucci, and E. Rajo-Iglesias, "On the increase of the efficiency and bandwidth of compact PIFAs based on SRR by making use of lumped capacitors," in Proc. IEEE APSURSI, 2010, pp. 1-4.

[15] E. Rajo-Iglesias, G. Villaseca-Sanchez, and C. Martin-Pascual, "Input impedance behavior in offset stacked patches," IEEE Antennas Wireless Propag. Lett., vol. 1, pp. 28-30, 2002.

[16] “CST Microwave Studio, User Manual Version 5.0," CST GmbH, Darmstadt, Germany.

[17] K. Rosengren, P.-S. Kildal, C. Carlsson, and J. Carlsson, "Characterization of antennas for mobile and wireless terminals in reverberation chambers: Improved accuracy by platform stirring," Microw. Opt. Technol. Lett., vol. 30, no. 20, pp. 391-397, Sep. 2001. 